Elect. Comm. in Probab. 13 (2008), 165-174

\title{
TIGHTNESS OF VOTER MODEL INTERFACES
}

\author{
ANJA STURM $^{1}$ \\ Department of Mathematical Sciences, University of Delaware, 501 Ewing Hall, Newark, DE \\ 19716-2553, USA \\ email: sturm@math.udel.edu
}

JAN M. SWART ${ }^{2}$

ÚTIA, Pod vodárenskou věži 4, 18208 Praha 8, Czech Republic

email: swart@utia.cas.cz

Submitted January 22, 2008, accepted in final form February 25, 2008

AMS 2000 Subject classification: Primary: 82C22; Secondary: 82C24, 82C41, 60K35.

Keywords: Long range voter model, swapping voter model, interface tightness, exclusion process.

\section{Abstract}

Consider a long-range, one-dimensional voter model started with all zeroes on the negative integers and all ones on the positive integers. If the process obtained by identifying states that are translations of each other is positively recurrent, then it is said that the voter model exhibits interface tightness. In 1995, Cox and Durrett proved that one-dimensional voter models exhibit interface tightness if their infection rates have a finite third moment. Recently, Belhaouari, Mountford, and Valle have improved this by showing that a finite second moment suffices. The present paper gives a new short proof of this fact. We also prove interface tightness for a long range swapping voter model, which has a mixture of long range voter model and exclusion process dynamics.

\section{Introduction and main results}

Let $X=\left(X_{t}\right)_{t \geq 0}$ be a long-range, one-dimensional 'swapping' voter model, i.e. $X$ is a Markov process with state space $\{0,1\}^{\mathbb{Z}}$ and formal generator $G:=G^{\mathrm{v}}+G^{\mathrm{s}}$, where

$$
\begin{aligned}
G^{\mathrm{v}} f(x) & :=\sum_{i j} q(i-j) 1_{\{x(i) \neq x(j)\}}\left\{f\left(x^{\{i\}}\right)-f(x)\right\}, \\
G^{\mathrm{s}} f(x) & :=\frac{1}{2} \sum_{i j} p(i-j) 1_{\{x(i) \neq x(j)\}}\left\{f\left(x^{\{i, j\}}\right)-f(x)\right\},
\end{aligned}
$$

\footnotetext{
${ }^{1}$ WORK SPONSORED BY UDRF GRANT 06000596 AND NSF GRANT 0706713

${ }^{2}$ WORK SPONSORED BY GA ČR GRANTS 201/06/1323 AND 201/07/0237
} 
$q$ and $p$ are functions $\mathbb{Z} \rightarrow[0, \infty)$, and $p$ is symmetric, i.e., $p(i)=p(-i)(i \in \mathbb{Z})$. Here, for any $x \in\{0,1\}^{\mathbb{Z}}$ and $\Delta \subset \mathbb{Z}$,

$$
x^{\Delta}(i):= \begin{cases}1-x(i) & \text { if } i \in \Delta, \\ x(i) & \text { if } i \notin \Delta\end{cases}
$$

denotes the configuration obtained from $x$ by flipping all spins in $\Delta$. Note that (1.1) says that if $X_{t}(i) \neq X_{t}(j)$ for some $i \neq j$, then due to the action of $G^{\mathrm{v}}, X_{t}(i)$ adopts the type of $X_{t}(j)$ with infection rate $q(i-j)$. This describes a long-range, one-dimensional voter model. In addition, due to the action of $G^{\mathrm{s}}$, at swapping rate $p(i-j)$ the sites $i$ and $j$ swap their types. In order for the process to be well-defined (see [Lig85]), we assume that $\sum_{i}(q(i)+p(i))<\infty$. We will also require irreducibility conditions. We call a rate function $r: \mathbb{Z} \rightarrow[0, \infty)$ irreducible if each $i \in \mathbb{Z}$ can be written as $i=i_{1}+\cdots+i_{n}$ with $n \geq 0$ and $r\left(i_{k}\right)>0$ for all $1 \leq k \leq n$. Consider the space

$$
S_{\mathrm{int}}:=\left\{x \in\{0,1\}^{\mathbb{Z}}: \lim _{i \rightarrow-\infty} x(i)=0, \lim _{i \rightarrow \infty} x(i)=1\right\}
$$

of states describing the interface between two infinite population of zeroes and ones. If the infection and swapping rates have a finite first moment, i.e., $\sum_{i}|i|(q(i)+p(i))<\infty$, then $X_{0} \in S_{\text {int }}$ implies $X_{t} \in S_{\text {int }}$ for all $t \geq 0$, a.s. (see [BMV07] for this statement concerning $G^{\mathrm{v}}$ ). Define an equivalence relation on $S_{\text {int }}$ by setting $x \sim y$ if $x$ and $y$ are translations of each other and let $\tilde{S}_{\text {int }}:=\left\{\tilde{x}: x \in S_{\text {int }}\right\}$, with $\tilde{x}:=\left\{y \in S_{\text {int }}: y \sim x\right\}$, denote the set of equivalence classes. Then $\tilde{X}=\left(\tilde{X}_{t}\right)_{t \geq 0}$ is a continuous-time Markov process with countable state space $\tilde{S}_{\text {int }}$. Let $x_{\mathrm{H}}(i):=1_{\{i \geq 0\}}$ denote the 'Heaviside state'. As long as $q(i)>0$ for some $i \neq 0$, it is not hard to see that $\tilde{x}_{\mathrm{H}}$ can be reached from any state in $\tilde{S}_{\text {int }}$, hence $\tilde{X}$ is an irreducible Markov process on the set of all states that can be reached from $\tilde{x}_{\mathrm{H}}$. Following Cox and Durrett [CD95], we say that $X$ exhibits interface tightness if $\tilde{X}$ is positively recurrent on this set.

Theorem 4 in [CD95] states that long-range voter models (without swapping) exhibit interface tightness provided that their infection rates $q$ are symmetric, irreducible, and have a finite third moment. Although not carried out there, their proofs also work if $q$ is asymmetric and $q_{\mathrm{s}}$ is irreducible, where $q_{\mathrm{s}}(i):=\frac{1}{2}(q(i)+q(-i))$ denote the symmetrized infection rates. Recently, Belhaouari, Mountford, and Valle [BMV07] improved this result by showing that a finite second moment suffices. They showed that this condition is sharp in the sense that interface tightness does not hold if $\sum_{i}|i|^{c} q(i)=\infty$ for some $c<2$. We will give a new, short proof of their sufficiency result. In fact, we will prove more:

\section{Theorem 1. (Interface tightness for long-range swapping voter models)}

Assume that $\sum_{i}|i|^{2}(q(i)+p(i))<\infty, q(i)>0$ for some $i \neq 0$, and that $q_{\mathrm{s}}+p$ is irreducible. Then $X$ exhibits interface tightness.

For the symmetric nearest-neighbor case, Theorem 1 has been proved in [BFMP01, Theorem 1.1 (ii) (b)]. They also have results for asymmetric exclusion dynamics (but symmetric voter dynamics). We note that the symmetric nearest-neighbor swapping voter model arises as the dual and interface model of certain systems of parity preserving branching and annihilating random walks, see [SS08, Section 2.1] and references there. Finally, we mention that for longrange voter models, the position of the interface, diffusively rescaled, converges to a Brownian motion with a known diffusion speed. In [BMSV06], it is shown that for this convergence, a finite $(3+\varepsilon)$-th moment of the infection rates is sufficient and a finite $(3-\varepsilon)$-th moment is necessary. That paper also contains sharp estimates for the tail distribution of the width of the interface. 


\section{Proof of Theorem 1}

The main idea of the proof is the same as in [CD95], namely, to look at the number of 'inversions':

$$
f_{\mathrm{CD}}(x):=\left|\left\{(i, j) \in \mathbb{Z}^{2}, i<j, x(i)>x(j)\right\}\right| \quad\left(x \in S_{\mathrm{int}}\right) .
$$

In [CD95, Section 4] and [BMV07], this quantity is estimated using duality and (subtle) results about one-dimensional random walk. Our approach will be to insert $f_{\mathrm{CD}}$ into the generator $G$ and prove that if interface tightness would not hold, then $f_{\mathrm{CD}}$ would decrease unboundedly as time tends to infinity, which yields a contradiction. In a way, our proof is similar to the methods in [BFMP01], which are based on Lyapunov functions. The function $f_{\mathrm{CD}}$, however, is not a Lyapunov function for our processs, so our proofs need a probabilistic ingredient as well, which is provided by Proposition 4 below.

We start by calculating $G f_{\mathrm{CD}}$.

Lemma 2. (Changes in number of inversions) For each $x \in S_{\mathrm{int}}$, one has

$$
G f_{\mathrm{CD}}(x)=\sum_{n=1}^{\infty}\left(q_{\mathrm{s}}(n)+p(n)\right) n^{2}-\sum_{n=1}^{\infty} q_{\mathrm{s}}(n) I_{n}(x),
$$

where

$$
I_{n}(x):=|\{i \in \mathbb{Z}: x(i) \neq x(i+n)\}| \quad(n \geq 1) .
$$

We will need the following lemma:

Lemma 3. (Nonnegative expectation) Assume that $\sum_{i}|i|^{2}(q(i)+p(i))<\infty$. Then

$$
\mathbb{E}\left[f_{\mathrm{CD}}\left(X_{0}\right)\right]+\int_{0}^{t} \mathbb{E}\left[G f_{\mathrm{CD}}\left(X_{s}\right)\right] \mathrm{d} s \geq 0 \quad(t \geq 0) .
$$

We will also need a result stating that the number of 'boundaries' between zeroes and ones, defined in the sense of (2.3), grows over time if interface tightness does not hold.

Proposition 4. (Interface growth) Assume that $\sum_{i}|i|(q(i)+p(i))<\infty, q(i)>0$ for some $i \neq 0$, and $q_{\mathrm{s}}+p$ is irreducible. Assume that interface tightness does not hold. Then

$$
\lim _{T \rightarrow \infty} \frac{1}{T} \int_{0}^{T} \mathrm{~d} t \mathbb{P}\left[I_{n}\left(X_{t}\right)<N\right]=0 \quad(N, n \geq 1) .
$$

With these statements we are now in the position to prove Theorem 1. Assume that interface tightness does not hold. By our assumptions on $q$ and $p$ we can choose $i, N \geq 1$ such that

$$
\sum_{n=1}^{\infty}\left(q_{\mathrm{s}}(n)+p(n)\right) n^{2}<q_{\mathrm{s}}(i) N .
$$


Then, by Lemmas 3 and 2 , and Proposition 4 , the process started in $X_{0}=x_{\mathrm{H}}$ satisfies

$$
\begin{aligned}
0 & \leq \int_{0}^{T} \mathrm{~d} t \mathbb{E}\left[G f_{\mathrm{CD}}\left(X_{t}\right)\right] \\
& =T \sum_{n=1}^{\infty}\left(q_{\mathrm{s}}(n)+p(n)\right) n^{2}-\sum_{n=1}^{\infty} q_{\mathrm{s}}(n) \int_{0}^{T} \mathrm{~d} t \mathbb{E}\left[I_{n}\left(X_{t}\right)\right] \\
& \leq T \sum_{n=1}^{\infty}\left(q_{\mathrm{s}}(n)+p(n)\right) n^{2}-q_{\mathrm{s}}(i) N \int_{0}^{T} \mathrm{~d} t \mathbb{P}\left[I_{i}\left(X_{t}\right) \geq N\right] \\
& =T \sum_{n=1}^{\infty}\left(q_{\mathrm{s}}(n)+p(n)\right) n^{2}-(T-o(T)) q_{\mathrm{s}}(i) N,
\end{aligned}
$$

as $T \rightarrow \infty$. Due to $(2.6)$ the right-hand side of $(2.7)$ tends to $-\infty$ as $T \rightarrow \infty$, which yields a contradiction.

\section{Proof of Lemmas 2 and 3, and Proposition 4}

Proof of Lemma 2 We need to count the number of pairs of sites $i, j$ with $i<j$ and $x(i)>x(j)$ that are created and deleted due to the various possible jumps. We will first consider $G^{\mathrm{s}} f_{\mathrm{CD}}$, i.e. the changes due to swapping. So consider the case that $x(i) \neq x(i+n)$ for some $n \in \mathbb{N}$, while there are $l$ ones on the left of $i, r$ zeroes on the right of $i+n$, and $n_{0}$ zeroes and $n_{1}$ ones between $i$ and $i+n$. Then the changes in $f_{\mathrm{CD}}(x)$ due to swapping can be summarized as follows:

$$
\begin{aligned}
& \underbrace{\ldots}_{l \times 1} 0 \underbrace{\ldots}_{n_{0} \times 0, n_{1} \times 1} 1 \underbrace{\ldots}_{r \times 0} \rightarrow \ldots 1 \ldots 0 \ldots \quad n_{0}+n_{1}+1=n \\
& \underbrace{\ldots}_{l \times 1} 1 \underbrace{\ldots}_{n_{0} \times 0, n_{1} \times 1} 0 \underbrace{\ldots}_{r \times 0} \rightarrow \ldots 0 \ldots 1 \ldots \quad-\left(n_{0}+n_{1}+1\right)=-n
\end{aligned}
$$

Thus, if we define

$$
I_{n}^{a b}(x):=|\{i: x(i)=a, x(i+n)=b\}| \quad(n \geq 0, a b=01,10)
$$

then we obtain

$$
G^{\mathrm{s}} f_{\mathrm{CD}}(x)=\sum_{n=1}^{\infty} p(n)\left(n \cdot I_{n}^{01}(x)-n \cdot I_{n}^{10}(x)\right)
$$

Now, for any $0 \leq m<n$, set

$$
I_{n, m}^{a b}(x):=|\{r \in \mathbb{Z}: x(n r+m)=a, x(n(r+1)+m)=b\}| .
$$

Walking along the thinned-out lattice $n \mathbb{Z}+m$ from $-\infty$ to $+\infty$, we see one more change from 0 to 1 than we see changes from 1 to 0 , i.e., $I_{n, m}^{01}(x)=I_{n, m}^{10}(x)+1$ for all $x \in S_{\text {int }}$. Since $I_{n}^{a b}(x)=\sum_{m=0}^{n-1} I_{n, m}^{a b}(x)$, it follows that

$$
I_{n}^{01}(x)=I_{n}^{10}(x)+n \quad\left(x \in S_{\mathrm{int}}\right) .
$$


This implies that (3.3) simplifies to

$$
G^{\mathrm{s}} f_{\mathrm{CD}}(x)=\sum_{n=1}^{\infty} p(n) n^{2}
$$

In order to consider the effect of $G^{\mathrm{v}}$ on $f_{\mathrm{CD}}$ we write

$$
\begin{aligned}
G^{\mathrm{v}} f_{\mathrm{CD}}(x)= & \sum_{k} q(-k)\left(\sum_{i: x(i)=1} \sum_{j: j>i}\left(1_{\{x(j)=1, x(j+k)=0\}}-1_{\{x(j)=0, x(j+k)=1\}}\right)\right. \\
& \left.+\sum_{i: x(i)=0} \sum_{j: j<i}\left(1_{\{x(j)=0, x(j+k)=1\}}-1_{\{x(j)=1, x(j+k)=0\}}\right)\right) .
\end{aligned}
$$

We observe that for any $x \in S_{\text {int }}$ and $k>0$,

$$
\begin{aligned}
& \sum_{j: j>i}\left(1_{\{x(j)=1, x(j+k)=0\}}-1_{\{x(j)=0, x(j+k)=1\}}\right) \\
& =\sum_{j=i+1}^{i+k} \sum_{n \geq 0}\left(1_{\{x(j+n k)=1, x(j+(n+1) k)=0\}}-1_{\{x(j+n k)=0, x(j+(n+1) k)=1\}}\right) \\
& =-\sum_{j=i+1}^{i+k} 1_{\{x(j)=0\}} .
\end{aligned}
$$

To see why the last equality in (3.8) holds, observe that since $k>0$, the sequence $x(j), x(j+$ $k), x(j+2 k) \ldots$ is eventually one. Hence, if $x(j)=1$, then the number of changes from 0 to 1 equals the number of changes from 1 to 0 and all terms cancel, while if $x(j)=0$ there is one extra change from 0 to 1 , leading to a contribution of minus one.

Likewise, for any $x \in S_{\text {int }}$ and $k>0$,

$$
\begin{aligned}
& \sum_{j: j<i}\left(1_{\{x(j)=0, x(j+k)=1\}}-1_{\{x(j)=1, x(j+k)=0\}}\right) \\
& =\sum_{j=i-k}^{i-1} \sum_{n \geq 0}\left(1_{\{x(j-n k)=0, x(j-(n-1) k)=1\}}-1_{\{x(j-n k)=1, x(j-(n-1) k)=0\}}\right) \\
& =\sum_{j=i}^{i+k-1} \sum_{n \geq 0}\left(1_{\{x(j-(n+1) k)=0, x(j-n k)=1\}}-1_{\{x(j-(n+1) k)=1, x(j-n k)=0\}}\right) \\
& =\sum_{j=i}^{i+k-1} 1_{\{x(j)=1\}} .
\end{aligned}
$$

It follows that for any $k>0$,

$$
\begin{aligned}
& \sum_{i: x(i)=1} \sum_{j: j>i}\left(1_{\{x(j)=1, x(j+k)=0\}}-1_{\{x(j)=0, x(j+k)=1\}}\right) \\
& +\sum_{i: x(i)=0} \sum_{j: j<i}\left(1_{\{x(j)=0, x(j+k)=1\}}-1_{\{x(j)=1, x(j+k)=0\}}\right) \\
& =-\sum_{i} \sum_{j=i+1}^{i+k} 1_{\{x(i)=1, x(j)=0\}}+\sum_{i} \sum_{j=i}^{i+k-1} 1_{\{x(i)=0, x(j)=1\}} \\
& =\sum_{n=1}^{k-1} I_{n}^{01}(x)-\sum_{n=1}^{k} I_{n}^{10}(x),
\end{aligned}
$$


Using (3.5), the expression in (3.10) can be rewritten as

$$
-I_{k}^{10}(x)+\sum_{n=1}^{k-1}\left(I_{n}^{01}(x)-I_{n}^{10}(x)\right)=-\frac{1}{2}\left(I_{k}(x)-k\right)+\sum_{n=1}^{k-1} n=\frac{1}{2}\left(k^{2}-I_{k}(x)\right),
$$

which holds for $k>0$. Using symmetry with respect to the map $x \mapsto x^{\prime}$ where $x^{\prime}(i):=$ $1-x(-i)$, it is not hard to see that we get the same formula for the expression in (3.10) if $k<0$. Inserting this into (3.7), we arrive at

$$
G^{\mathrm{v}} f_{\mathrm{CD}}(x)=\sum_{n=1}^{\infty} q_{\mathrm{s}}(n)\left(n^{2}-I_{n}(x)\right)
$$

Taking (3.6) and (3.12) together now implies (2.2).

Proof of Lemma 3 If the function $f_{\mathrm{CD}}$ were bounded, then standard theory would tell us that the process

$$
M_{t}:=f_{\mathrm{CD}}\left(X_{t}\right)-\int_{0}^{t} G f_{\mathrm{CD}}\left(X_{s}\right) \mathrm{d} s \quad(t \geq 0)
$$

is a martingale with respect to the filtration generated by $X$. In particular, since $\mathbb{E}\left[M_{t}\right]=$ $\mathbb{E}\left[M_{0}\right]$ and $f_{\mathrm{CD}} \geq 0$, this would imply (2.4). In the present case, since $f_{\mathrm{CD}}$ is unbounded, we have to work a bit. Let

$$
w(x):=\max \{i: x(i) \neq x(i+1)\}-\min \{i: x(i) \neq x(i+1)\}
$$

denote the 'width' of an interface state $x \in S_{\text {int }}$. We can couple $X_{t}$ to a continuous-time random walk $\left(R_{t}\right)_{t \geq 0}$, started in $R_{0}=w\left(X_{0}\right)$, which jumps from $r$ to $r+n$ with rate

$$
a(n):=\sum_{k=n}^{\infty} 2\left(q_{\mathrm{s}}(k)+p(k)\right)
$$

in such a way that $R_{t} \geq w\left(X_{t}\right)$ for all $t \geq 0$ a.s. We check that

$$
\begin{aligned}
& \sum_{n=1}^{\infty} a(n) n=\sum_{n=1}^{\infty} \sum_{k=n}^{\infty} 2\left(q_{\mathrm{s}}(k)+p(k)\right) n=\sum_{k=1}^{\infty} \sum_{n=1}^{k} 2\left(q_{\mathrm{s}}(k)+p(k)\right) n \\
& \quad=\sum_{k=1}^{\infty} 2\left(q_{\mathrm{s}}(k)+p(k)\right) \frac{1}{2} k(k+1)<\infty
\end{aligned}
$$

Let $X^{K}$ be the process with generator given by

$$
\begin{aligned}
G^{K} f(x):= & \sum_{i j:|i-j| \leq K} q(i-j) 1_{\{x(i) \neq x(j)\}}\left\{f\left(x^{\{i\}}\right)-f(x)\right\}, \\
& +\frac{1}{2} \sum_{i j:|i-j| \leq K} p(i-j) 1_{\{x(i) \neq x(j)\}}\left\{f\left(x^{\{i, j\}}\right)-f(x)\right\},
\end{aligned}
$$

started in $X_{0}^{K}=X_{0}$. We set

$$
\tau_{K, N}:=\inf \left\{t \geq 0: w\left(X_{t}^{K}\right)>N\right\}
$$


and define $\tau_{N}$ similarly, with $X^{K}$ replaced by $X$. It follows from standard theory that

$$
M_{t}^{K, N}:=f_{\mathrm{CD}}\left(X_{t \wedge \tau_{K, N}}^{K}\right)-\int_{0}^{t \wedge \tau_{K, N}} G^{K} f_{\mathrm{CD}}\left(X_{s}^{K}\right) \mathrm{d} s \quad(t \geq 0)
$$

is a martingale, where by Lemma 2 ,

$$
G^{K} f_{\mathrm{CD}}(x)=\sum_{n=1}^{K}\left(q_{\mathrm{s}}(n)+p(n)\right) n^{2}-\sum_{n=1}^{K} q_{\mathrm{s}}(n) I_{n}(x) .
$$

Using an obvious coupling, we may arrange that $X^{K}$ converges a.s. to $X$ in such a way that there exists a random $K_{0}$ such that for all $K \geq K_{0}$, one has $\tau_{K, N}=\tau_{N}$ and $X_{t}^{K}=X_{t}$ for all $t \leq \tau_{N}$. Since moreover $\tau_{N} \rightarrow \infty$ as $N \rightarrow \infty$ (which follows from the estimate $w\left(X_{t}\right) \leq R_{t}$ ), taking into account (2.2) and (3.20), it follows that

$$
\lim _{N \rightarrow \infty} \lim _{K \rightarrow \infty} \int_{0}^{t \wedge \tau_{K, N}} G^{K} f_{\mathrm{CD}}\left(X_{s}^{K}\right) \mathrm{d} s=\int_{0}^{t} G f_{\mathrm{CD}}\left(X_{s}\right) \mathrm{d} s \quad \text { a.s. }
$$

Since $M^{K, N}$ is a martingale,

$$
0 \leq \mathbb{E}\left[f_{\mathrm{CD}}\left(X_{t \wedge \tau_{K, N}}^{K}\right)\right]=\mathbb{E}\left[f_{\mathrm{CD}}\left(X_{0}\right)\right]+\mathbb{E}\left[\int_{0}^{t \wedge \tau_{K, N}} G^{K} f_{\mathrm{CD}}\left(X_{s}^{K}\right) \mathrm{d} s\right] .
$$

Letting $K \rightarrow \infty$ and then $N \rightarrow \infty$ in (3.22), using (3.21), we arrive at (2.4), provided we show (in view of $(3.20)$ ) that the random variables

$$
\sum_{n=1}^{K} q_{\mathrm{s}}(n) \int_{0}^{t \wedge \tau_{K, N}} I_{n}\left(X_{s}^{K}\right) \mathrm{d} s \quad(K, N \geq 1)
$$

are uniformly integrable. We can couple the $X^{K}$ to a random walk $\left(R_{t}\right)_{t \geq 0}$ with jump rates $a(n)$ as in (3.15), in such a way that $R_{t} \geq w\left(X_{t}^{K}\right)$ for all $t \geq 0$ and $K \geq 1$ a.s. Hence, since $I_{n}(x) \leq n+w(x)$, we may estimate

$$
\sum_{n=1}^{K} q_{\mathrm{s}}(n) \int_{0}^{t \wedge \tau_{K, N}} I_{n}\left(X_{s}^{K}\right) \mathrm{d} s \leq \sum_{n=1}^{\infty} q_{\mathrm{s}}(n) \int_{0}^{t}\left(n+R_{s}\right) \mathrm{d} s .
$$

By (3.16), the right-hand side of (3.24) has finite expectation, proving the required uniform integrability.

Remark 5. (Martingale problem) If $\sum_{i}|i|^{3}(q(i)+p(i))<\infty$, then the jump rates $a(n)$ in (3.15) have a finite second moment. Using this and the estimate $f_{\mathrm{CD}}(x) \leq w(x)^{2}$, one can prove that in this case the process in (3.13) is a martingale.

Proof of Proposition 4 Consider the 'boundary process'

$$
Y_{t}(i):=1_{\left\{X_{t}(i) \neq X_{t}(i+1)\right\}} \quad(t \geq 0, i \in \mathbb{Z}),
$$

which is a Markov process in

$$
S_{\text {bound }}:=\left\{y \in\{0,1\}^{\mathbb{Z}}: \sum_{i} y(i) \text { is finite and odd }\right\} .
$$


Then $\left(Y_{t}\right)_{t \geq 0}$ is a Markov process in $\{0,1\}^{\mathbb{Z}}$ with formal generator

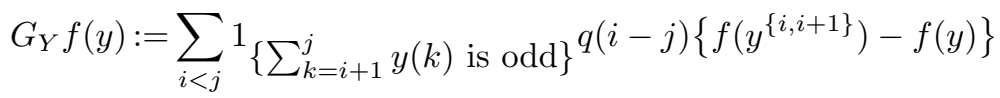

$$
\begin{aligned}
& \left.+\sum_{i>j} 1_{\left\{\sum_{k=j+1}^{i}\right.} y(k) \text { is odd }\right\}^{q(i-j)\left\{f\left(y^{\{i, i+1\}}\right)-f(y)\right\}} \\
& +\sum_{i<j-1} 1_{\left\{\sum_{k=i+1}^{j} y(k) \text { is odd }\right\}} p(i-j)\left\{f\left(y^{\{i, i+1, j, j+1\}}\right)-f(y)\right\} \\
& +\sum_{i} 1_{\{y(i+1)=1\}} p(1)\left\{f\left(y^{\{i, i+2\}}\right)-f(y)\right\} \text {. }
\end{aligned}
$$

Using the fact that $\sum_{i}|i|(q(i)+p(i))<\infty$, one can check that $Y$ is a parity preserving cancellative spin system in the sense of [SS08]. We will show that the process started in $y$, denoted by $Y^{y}$, satisfies

$$
\inf \left\{\mathbb{P}\left[\left|Y_{t}^{y}\right|=n\right]:|y|=n+2, y(i)=1=y(j) \text { for some } i \neq j,|i-j| \leq L\right\}>0
$$

$(L \geq 1, n \geq 0, t>0)$. As a result of (3.28), we can apply [SS08, Proposition 13] to conclude that

$$
\lim _{T \rightarrow \infty} \frac{1}{T} \int_{0}^{T} \mathrm{~d} t \mathbb{P}\left[I_{1}\left(X_{t}\right)<N\right]=0 \quad(N \geq 1) .
$$

See also Proposition 2.6 in [Han99] for similar arguments concerning a dual process to the threshold voter model.

To give a rough idea of the proof and of the importance of condition (3.28), think of the sites with $Y_{t}(i)=1$ as being occupied by a particle. Then $Y$ is a parity preserving particle system, i.e., if $Y$ is started in an odd (even) initial state, then the number of particles always stays odd (even). Let $\tilde{Y}$ denote the process obtained from $Y$ by identifying states that are a translation of each other. If $\tilde{X}$ is not positively recurrent, then the same is true for $\tilde{Y}$. The main idea of the proof of (3.29) is to use induction on $n$ to show that there cannot be less than $n$ particles for a positive fraction of time. This is obviously true for $n=1$; imagine that it holds for a certain $n$. If we see $n$ particles for a positive fraction of time, then most of the time these particles must be situated far from each other, for else with positive probability at least two would annihilate each other due to (3.28), violating the induction hypothesis. However, since $\tilde{Y}$ is not positively recurrent, $n$ single particles far from each other will soon each produce three particles, hence we cannot see $n$ particles for a positive fraction of time.

To boost up (3.29) to the statement in (2.5), it suffices to show that the process started in $x$, denoted by $X^{x}$, satisfies

$$
\lim _{N \rightarrow \infty} \inf _{\left|I_{1}(x)\right| \geq N} \mathbb{P}\left[I_{n}\left(X_{t}^{x}\right)<M\right]=0 \quad(M, n \geq 1, t>0) .
$$

For if (3.30) holds, then for each $t, \varepsilon>0$ we can choose $N$ large enough such that the limit in (3.30) is smaller than $\varepsilon$, and therefore, by (3.29) and a restart argument

$$
\limsup _{T \rightarrow \infty} \frac{1}{T} \int_{t}^{t+T} \mathrm{~d} s \mathbb{P}\left[I_{n}\left(X_{s}\right)<M\right] \leq \varepsilon \quad(M \geq 1) .
$$

Since $\varepsilon>0$ is arbitrary, this implies $(2.5)$.

We still need to prove $(3.28)$ and (3.30). We start with the former. Choose $k \neq 0$ such that $q(k)>0$. By symmetry, we may without loss of generality assume that $k>0$. It suffices to 
prove (3.28) for $L \geq k$. So fix $L \geq k, n \geq 0$, and $t>0$. Let $y=\delta_{i_{1}}+\cdots+\delta_{i_{n}}$ where $\delta_{i}(j):=1$ if $i=j$ and $=0$ otherwise, and $i_{1}<\cdots<i_{n}$. Assume that

$$
M:=\left\{m^{\prime} \in\{1, \ldots, n-1\}: i_{m^{\prime}+1}-i_{m^{\prime}} \leq L\right\}
$$

is not empty and let $m:=\inf (M)$. Then $x\left(i_{m}+1-L\right)=\cdots=x\left(i_{m}\right) \neq x\left(i_{m}+1\right)$, and hence, since $L \geq k$, there is a positive probability that during the time interval $[0, t]$, the sites $i_{m}+1, \ldots, i_{m+1}$ get infected successively by the sites $i_{m}+1-k, \ldots, i_{m+1}-k$, leading to a decrease in $I_{1}\left(X_{t}\right)$ of 2 . Using the fact that $\sum_{i}|i|(q(i)+p(i))<\infty$, it is not hard to see that moreover, with positive probability, no other infections take place, and that this probability is uniformly bounded from below in all $y$ satisfying our assumptions.

To prove (3.30), we view the dynamics of our process $X$ as follows. For each ordered pair $(i, j)$ with $i \neq j$, at times selected according to an independent Poisson point process with intensity $q(i-j)$, the type of site $j$ infects the site $i$. Likewise, for each unordered pair $\{i, j\}$ with $i \neq j$, at times selected according to an independent Poisson point process with intensity $p(i-j)$, the sites $i$ and $j$ swap their types.

We claim that if we view the evolution of types in this way, then we can find a $j \in \mathbb{Z}$ such that with positive probability $X_{t}(j)$ inherits its type from $x(0)$ and $X_{t}(j+n)$ inherits its type from $x(1)$. To see this, we will make a number of infections and swaps to transport the type of site 0 to $j$ and the type of site 1 to $j+n$. Let us say that in the $k$-th step of our construction, we have transported the type of site 0 to the site $l_{k}$ and the type of site 1 to $r_{k}$. Then, in the $(k+1)$-th step of our construction, by making an infection with rate $q(i)$, we may transport the type of site $l_{k}$ to $l_{k}+i$ and set $\left(l_{k+1}, r_{k+1}\right):=\left(l_{k}+i, r_{k}\right)$, or we may transport the type of site $r_{k}$ to $r_{k}+i$ and set $\left(l_{k+1}, r_{k+1}\right):=\left(l_{k}, r_{k}+i\right)$, and similarly for swaps. Thus, in each step, we may increase $r_{k}-l_{k}$ by $d$ for each $d \in \mathcal{G}:=\left\{i \in \mathbb{Z}: q_{\mathrm{s}}(i)+p(i)>0\right\}$. We must only make sure that when we move the type of one site to a new position, we do not influence the type of the other site. To avoid this sort of influence, we will make sure that at each point in our construction $l_{k}<r_{k}$. We claim that this is possible. Set $n_{k}:=r_{k}-l_{k}$. We need to show that there exist (strictly) positive integers $n_{0}, \ldots, n_{m}$ such that $n_{0}=1, n_{m}=n$, and $\left(n_{k}-n_{k-1}\right) \in \mathcal{G}$ for all $1 \leq k \leq m$. By our assumption that $q_{\mathrm{s}}+p$ is irreducible we can write $n-1=i_{1}+\cdots+i_{m}$ with $i_{1}, \ldots, i_{m} \in \mathcal{G}$. (Note that this is the only place in our proofs where we use irreducibility.) Without loss of generality we may assume that $i_{1} \geq \cdots \geq i_{m}$. Then setting $n_{k}:=1+i_{1}+\cdots+i_{k}$ proves our claim.

It follows that there exist $j \in \mathbb{Z}$ and $L \geq j$ such that whenever $x(i) \neq x(i+1)$, there is a positive probability that $X_{t}(i+j)$ inherits its type from $x(i)$ and $X_{t}(i+j+n)$ inherits its type from $x(1)$ through a sequence of infections and swaps that are entirely contained in $\{i-L, \ldots, i+L\}$. If $I_{1}(x)$ is large, we can find many sites $i$, situated at least a distance $3 L$ from each other, such that $x(i) \neq x(i+1)$. By what we have just proved each pair has an independent probability to produce at time $t$ two sites $i+j$ and $i+j+n$ such that $X_{t}(i+j) \neq X_{t}(i+j+n)$, hence $I_{n}\left(X_{t}\right)$ is with large probability large.

Acknowledgement We thank Rongfeng Sun who found an error in our original statement of Lemma 3 and told us how to fix it. We thank the referee for bringing reference [BMSV06] to our attention.

\section{References}

[BFMP01] V. Belitsky, P.A. Ferrari, M.V. Menshikov, and S.Y. Popov. A mixture of the exclusion process and the voter model. Bernoulli 7(1): 119-144, 2001. Zbl 0978.60105. 


\section{MR1811747}

[BMSV06] S. Belhaouari, T. Mountford, R.Sun, and G. Valle. Convergence results and sharp estimates for the voter model interfaces. Electron. J. Probab. 11: Paper No. 30, 768-801, 2006. Zbl 1113.60092. MR2242663

[BMV07] S. Belhaouari, T. Mountford, and G. Valle. Tightness for the interfaces of onedimensional voter models. Proc. Lond. Math. Soc. (3) 94(2): 421-442, 2007. Zbl 1112.60074. MR2308233

[CD95] J.T. Cox and R. Durrett. Hybrid zones and voter model interfaces. Bernoulli 1(4): 343-370, 1995. Zbl 0849.60088. MR1369166

[Han99] S.J. Handjani. The complete convergence theorem for coexistent threshold voter models. Ann. Probab. 27(1): 226-245, 1999. Zbl 0974.60093. MR1681118

[Lig85] T.M. Liggett. Interacting Particle Systems. Springer, New York, 1985. Zbl 0559.60078. MR0776231

[SS08] A. Sturm and J.M. Swart. Voter models with heterozygosity selection. Ann. Appl. Probab 18(1): 59-99, 2008. MR2380891 